\title{
Pheochromocytoma presenting as hyperglycemic hyperosmolar syndrome and unusual fever
}

\author{
I-Shuan Lee • Ting-Wei Lee $\cdot$ Chun-Jen Chang • \\ Yu-Mei Chien · Ting-I Lee
}

Received: 16 January 2015/ Accepted: 15 February 2015/Published online: 3 March 2015

(C) SIMI 2015

\begin{abstract}
A 68-year-old woman was sent to the emergency department (ED) because of alterations in her level of consciousness. The patient had polydipsia and experienced a weight loss of about $6 \mathrm{~kg}$, which was associated with progressive general weakness over a period of several months. Intermittent diaphoresis and palpitation with occasional headache were also noted. She had no known underlying systemic disease, such as hypertension or cardiac dysrhythmia. She denied taking any medication. She smoked 0.2 packs per day for 3 years, but denied taking either alcohol or illicit drugs. She denied a family history of diabetes mellitus (DM) or any familial diseases.

At the ED, the patient's body mass index was underweight at $17.6 \mathrm{~kg} / \mathrm{m}^{2}$. Her body temperature was $38.0^{\circ} \mathrm{C}$, with a blood pressure of $199 / 118 \mathrm{mmHg}$, pulse rate 129 beats per minute, and respiratory rate 18 breaths per minute. The oxygen saturation was $93 \%$ while she was breathing in ambient air. The laboratory data showed a newly-diagnosed DM complicated with a hyperosmolar hyperglycemic state (serum glucose at $1836 \mathrm{mg} / \mathrm{dl}$, serum sodium $124 \mathrm{mmol} / \mathrm{l}$, hemoglobin $\mathrm{A}_{\mathrm{C}} 14.4 \%$, and effective osmolality $350 \mathrm{mOsm} / \mathrm{kg}$ ). After adequate hydration,
\end{abstract}

I.-S. Lee $\cdot$ T.-W. Lee $\cdot$ C.-J. Chang · Y.-M. Chien ·

T.-I. Lee $(\bowtie)$

Division of Endocrinology and Metabolism, Department of Internal Medicine, Wan Fang Hospital, Taipei Medical University, 111 Shin Lung Road Section 3, Taipei, Taiwan e-mail: aglee@ms29.hinet.net; agleems29@gmail.com

T.-W. Lee

Graduate Institute of Clinical Medicine, College of Medicine, Taipei Medical University, Taipei, Taiwan

T.-I. Lee

Department of General Medicine, College of Medicine,

Taipei Medical University, Taipei, Taiwan insulin therapy and appropriate antibiotics for suspected aspiration pneumonia, the patient's blood sugar improved and the fever subsided. During hospitalization, blood pressures were 100-120/60-70 mmHg without any sign of hypertension or signs suggestive of orthostatic changes.

However, on the 20th hospital day, the patient had a second bout of fever with sepsis-like presentation (high fever $39.3{ }^{\circ} \mathrm{C}$, tachycardia 110 beats per minute, tachypnea, but without leukocytosis) that was refractory to antibiotic therapy. Abdominal sonography was performed to search for the infection focus, which revealed an intraabdominal tumor near the liver and kidney. A subsequent computed tomography confirmed a 4-cm solid heterogeneous mass lesion in the right adrenal gland (Fig. 1a). Based on the patient's clinical presentations (markedly elevated blood pressure at ED, history of intermittent palpitation, and unusual fever), a pheochromocytoma was highly likely. Collection of 24-h urine for catecholamine and vanillylmandelic acid (VMA) levels showed the isolated elevation of epinephrine levels. Repeated collection of urinary examination for catecholamines and VMA levels were performed after the patient was free from her acute illness showed similar results (Table 1). Pre-operative treatment with $\alpha$ - and $\beta$-blockade treatment were given to the patient, and she underwent uneventful laparoscopic right adrenalectomy. The pathological examination confirmed our diagnosis of pheochromocytoma (Fig. 1b). The patient remained symptom-free with normal urinary catecholamines excretion after 6 months of post-operative follow-up. Her DM is presently under good glycemic control (hemoglobin $\mathrm{A} 1_{\mathrm{C}} 6.1 \%$ ) using an oral antidiabetic agent (metformin $250 \mathrm{mg}$ daily). Moreover, she gradually gained weight to a body mass index of $23.4 \mathrm{~kg} / \mathrm{m}^{2}$.

Pheochromocytoma is a rare catecholamine-producing tumor that most commonly presents with episodes of 


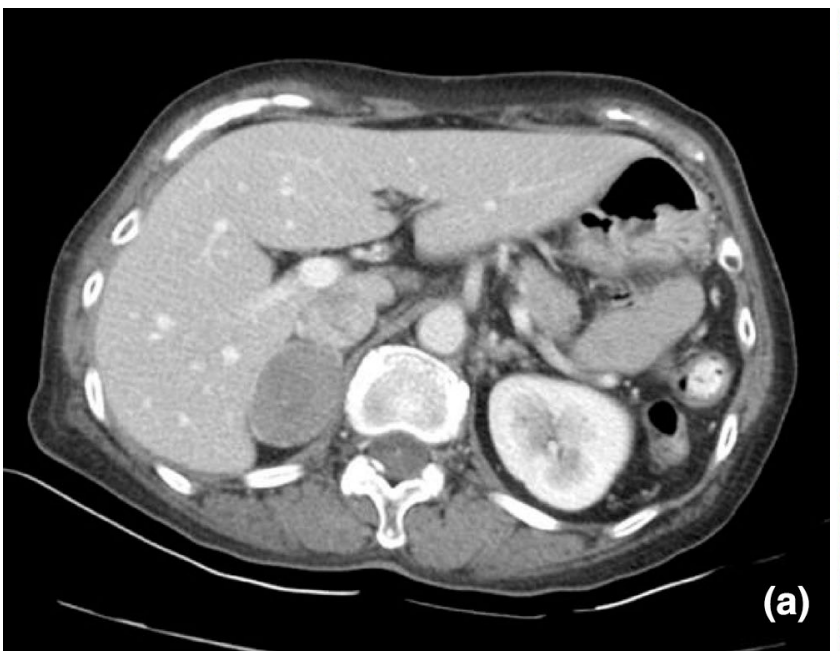

Fig. 1 Abdominal computed tomography shows a $4 \mathrm{~cm}$ solid heterogeneous mass lesion in the right adrenal gland with internal low attenuation foci. No regional adenopathy, vascular invasion or metastatic disease is evident (a). Pheochromocytoma cells stained

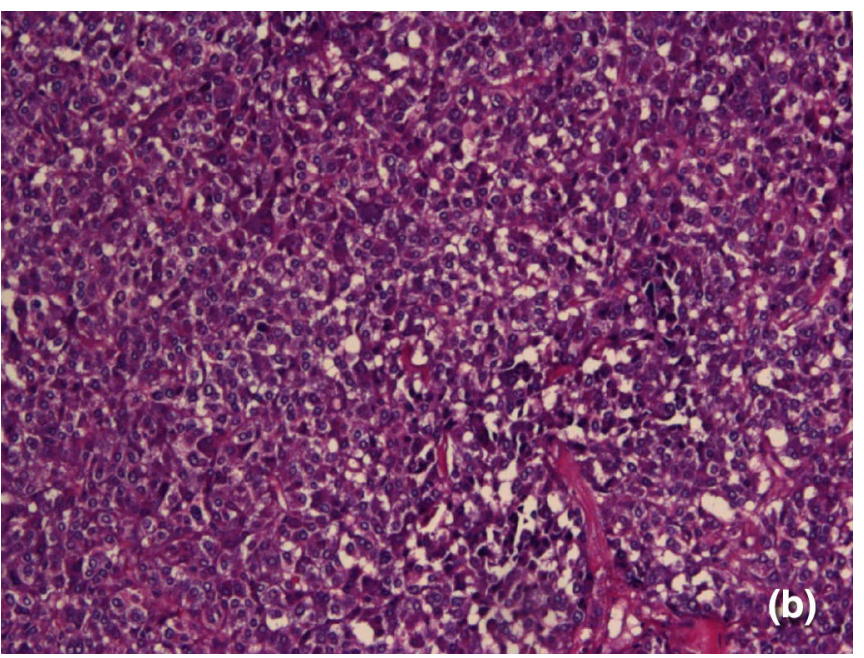

with hematoxylin and eosin stain, at $\times 100$ magnification under a microscope. The tumor cells have large round vesicular nuclei and abundant basophilic to amphophilic cytoplasm. Increased vascularity is noted. There is no increased mitotic count (b)

Table 1 Pre-operative and post-operative urinary catecholamine parameters

\begin{tabular}{|c|c|c|c|c|}
\hline 24-hour urinary excretion & During hospitalization & Free from acute illness & Post operation & Reference \\
\hline Epinephrine $(\mu \mathrm{g} /$ day $)$ & 247.4 & 162.2 & 6.2 & $<27$ \\
\hline Norepinephrine ( $\mu \mathrm{g} /$ day) & 35.9 & 70.7 & 44.4 & $<97$ \\
\hline Dopamine $(\mu \mathrm{g} /$ day $)$ & 166.1 & 310.6 & 271 & $<500$ \\
\hline Vanillylmandelic acid (mg/day) & 7.01 & 6.50 & - & $1.0-7.5$ \\
\hline 24- hour urine creatinine total volume (g/day) & 0.8 & - & 0.9 & $0.6-2$ \\
\hline Urine creatinine $(\mathrm{mg} / \mathrm{dl})$ & 62 & - & 48 & $30-125$ \\
\hline 24- hour urine total volume $(\mathrm{ml})$ & 1300 & 1780 & 1940 & \\
\hline
\end{tabular}

headaches, sweating, palpitations, and paroxysmal or sustained hypertension. However, clinical manifestations of pheochromocytoma are highly variable, and can mimic several other diseases, such as acute coronary syndrome, heart failure, and sepsis [1]. Paroxysmal or sustained hypertension is one of the major presentations of pheochromocytoma. However, excess catecholamine secretions in a patient with pheochromocytoma can also result in cardiovascular complications without hypertension [2]. Therefore, a timely diagnosis and treatment of pheochromocytoma is very important.

Different catecholamine secretions from pheochromocytoma have different effects on blood glucose and blood pressure levels [3]. About 40-50\% of patients with pheochromocytomas clinically present as glucose intolerance or DM, which are such common pathologic conditions that they can delay the timely diagnosis of pheochromocytoma. Hyperglycemia has been linked to aggravation of insulin resistance caused by catecholamine excess in pheochromocytoma. The inhibitory effect of catecholamines on insulin secretion is thought to be mediated by $\alpha-2$ adrenergic receptors. The isolated elevation of epinephrine levels in our case had a profound effect on glucose intolerance, inducing lipolysis and hepatic gluconeogenesis, but inhibited peripheral glucose utilization. About $54 \%$ of adrenergic producing tumors in the adrenal glands are diagnosed in patients of advanced age, similar to our case. The possible mechanism that led to our patient's presenting with DM complicated with hyperosmolar hyperglycemic state may have been that the isolated catecholamine excess with epinephrine secreting pheochromocytoma resulted in an increase in lipolysis, which reduced insulin secretion and increased glucose production from the liver. Although our patient, a few months prior to admission presented clinically with intermittent catecholamine excess that included intermittent diaphoresis, palpitation, and occasional headache, there was no evidence of hypertension found during admission. However, unlike the usual presentation 
where one would expect hypotension from volume depletion at the height of the hyperglycemic hyperosmolar state, she had marked hypertension. Additionally, DM patients complicated with hyperosmolar hyperglycemic state usually have impaired consciousness or have a bed-ridden status, which was not consistent with our patient. It is therefore important to consider pheochromocytoma as one of our differential diagnoses in an ambulatory patient presenting with marked hypertension at the height of hyperosmolar hyperglycemic state, especially when the clinical presentation is different from the usual signs and symptoms.

Although measurements of plasma-free metanephrines or urinary fractionated metanephrines are the most sensitive and most reliable indicators for the exclusion of pheochromocytoma, these were unavailable in our medical center [4]. In this case, only the traditional biochemical exams that include collection of 24-h urine catecholamines and VMA were performed. However, urine catecholamine concentrations increase during pathophysiological or pharmacological adrenosympathetic stimulation, which can lead to the misdiagnosis of pheochromocytomas. Additionally, these bioamines are also very sensitive to diets, medications and adrenosympathetic stimulation, making the interpretations of laboratory results unreliable in the diagnosis and monitoring of pheochromocytomas. For these reasons, a repeated collection of urine for catecholamines and VMA levels were performed when the patient recovered from her acute illness. The results showed persistent elevation of epinephrine levels, thus, supporting the diagnosis of an epinephrine-producing adrenal tumor. Although VMA has a high specificity rate, the VMA level in our case was an unreliable marker for pheochromocytoma.

On initial presentation, our patient was underweight, and this could have been due to chronic catecholamine overproduction caused by pheochromocytoma that may have led to the increase in proinflammatory cytokines and the hypermetabolic state characterized by increased resting energy expenditure. Adrenalectomy led to the normalization of the energy metabolism in our case followed by an increase in body mass index and body fat content with a decrease in inflammatory markers and cytokines [5]. It is therefore of utmost importance, to look for an undiscovered endocrinopathy with hypermetabolic state in cases of an underweight patient presenting with a hyperosmolar hyperglycemic state not having any previous history of DM.

Here we highlight the importance of integrating pheochromocytoma-complicating DM in a case presenting with hyperosmolar hyperglycemic state with marked hypertension and fever but without a focus of infection.

Conflict of interest Authors declare that they have no conflict of interest.

Statement of human and animal rights All procedures followed were in accordance with the ethical standards of the responsible committees (institutional and national) and with the Helsinki Declaration of 1975, as revised in 2008 .

Informed consent Informed consent was obtained from the participant for being included in this study according to Ethics Committees requirements.

\section{References}

1. Lee TW, Lin KH, Chang CJ, Lew WH, Lee TI (2013) Pheochromocytoma mimicking both acute coronary syndrome and sepsis: a case report. Med Princ Pract 22:405-407

2. Stolk RF, Bakx C, Mulder J, Timmers HJ, Lenders JW (2013) Is the excess cardiovascular morbidity in pheochromocytoma related to blood pressure or to catecholamines? J Clin Endocrinol Metab 98:1100-1106

3. Mannelli M, Lenders JW, Pacak K, Parenti G, Eisenhofer G (2012) Subclinical phaeochromocytoma. Best Pract Res Clin Endocrinol Metab 26:507-515

4. Lenders JW, Pacak K, Walther MM, Linehan WM, Mannelli M, Friberg P, Keiser HR, Goldstein DS, Eisenhofer G (2002) Biochemical diagnosis of pheochromocytoma: which test is best? JAMA 287:1427-1434

5. Petrak O, Haluzikova D, Kavalkova P, Strauch B, Rosa J, Holaj R, Brabcova Vrankova A, Michalsky D, Haluzik M, Zelinka T et al (2013) Changes in energy metabolism in pheochromocytoma. J Clin Endocrinol Metab 98:1651-1658 